\title{
Self-assembly Coloration Approach on Cotton Fibers using Porphyrin
}

\author{
Byung-Soon Kim, Xiaochuan Li, Sung-Hoon Kim, Jin-Seok Bae ${ }^{1}$ and Young-A Son ${ }^{\dagger}$
}

\author{
BK 21 FTIT, Dept. of Organic Materials and Textile System Engineering, \\ Chungnam National University, Daejeon, 305-764, South Korea \\ ${ }^{1}$ Dept. of Textile System Engineering, Kyungpook National University, Daegu, 702-701, South Korea
}

(Received: September 2, 2008/Revised: October 16, 2008/Accepted: October 21, 2008)

\begin{abstract}
In this work, poly(diallyldimethylammoniumchloride) (PDDAC) and meso-tetrakis(4-carboxyphenyl)porphyrin were considered to produce the self-assembly fabrication films. This method is based on the layer-by-layer (LbL) deposition produced by the electrostatic attraction between positively charged PDDAC and negatively charged porphyrin ions. The result of multilayer fabrication was discussed with the level of color strength $(\mathrm{K} / \mathrm{S}) . \mathrm{K} / \mathrm{S}$ spectra of the fabricated multilayer films showed gradual increase behaviors. In addition, the color photo images of the fabricated multilayer films showed that PDDAC and porphyrin were successfully attracted by electrostatic self-assembly forces.

Keywords: self-assembly, multilayer, fabrication, LbL poly(diallyldimethylammonium chloride), meso-tetrakis(4-carboxyphenyl) porphyrin
\end{abstract}

\section{Introduction}

Since the first reported by Decher, many efforts have been focused on the preparation of polyelectrolyte multilayer films based on electrostatic self-assembly layer-by-layer ( $\mathrm{LbL})$ technique $^{1-3}$. This $\mathrm{LbL}$ technique has been widely considered in many scientific and industrial areas due to their applicable potentials. The LbL technique presents easy and convenient way to control the construction of thin film layers ${ }^{4-7)}$. The $\mathrm{LbL}$ technique is designed on alternating electrostatic bindings of the +/- charged polyions. There is many academic research reports to explain the theory and principle of this experimental attempt.

Porphyrins $^{8-10)}$ are well announced to form useful aggregates caused by non-covalent bonding effects. The optical absorptional and photochemical properties are being regarded as an interesting factors of this material. In this context, porphyrin is one of the most widely used molecules in medicine, electronic, optical devices and sensors due to its excellent stabilities and unique optical and electronic properties ${ }^{11)}$.

Herein, the direct coloration approach using LbL application between meso-tetrakis(4-carboxyphenyl) porphyrin and cellulosic substrate was considered. This attempt may be a useful technique to produce coloration effects, in which the number of layers and corresponding color strength could be easily controlled and monitored.

To make a shape of the cationic surface layer, poly (diallyldimethylammonium chloride) (PDDAC) was firstly deposited on the cellulosic substrates. And porphyrin having negative charge was successively layered and fabricated. This application skill has been studied and presented in recent days from Tani and Nakamura ${ }^{12)}$. However, there are few paper publishment on LbL coloration approach to polymeric substrates.

Thus, the growth of fabrication layers on cotton fibers produced by LbL attraction was studied.

The level of porphyrin attraction was discussed with the color strength $(\mathrm{K} / \mathrm{S})$ values in detail.

Reflectance measurements on the substrate were carried out. 


\section{Experimental}

\subsection{Material}

Poly(diallyldimethylammonium chloride) (PDDAC) and $m e s o$-tetrakis(4-carboxyphenyl)porphyrin ${ }^{13)}$ were purchased from Aldrich. The doublely distilled water was used for preparing self-assembly fabrication layers. This water soluble porphyrin was chosen to meet the application purpose for self-assembly electrostatic layerby-layer technique in aqueous medium. Structures of PDDAC and porphyrin are shown in Fig. 1 (a) and (b), respectively. UV-Vis absorption spectra of PDDAC and porphyrin in water are also shown in Fig. 2.

\subsection{Fabrication of self-assembly multilayer}

The self-assembly multilayer fabrication process between PDDAC and porphyrin was prepared by alternatively immersing the cotton fibers into PDDAC and porphyrin aqueous solution with intermediate washing using Milli-Q-water. In the negatively charged porphyrin aqueous solution, $0.1 \mathrm{~g}$ of $\mathrm{NaOH}$ was added to assist the solubilitv in distilled water.

(a)

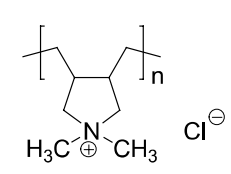

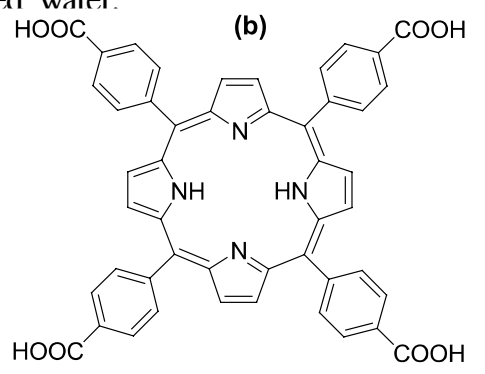

Fig. 1. Structures of poly(diallyldimethylammonium chloride)

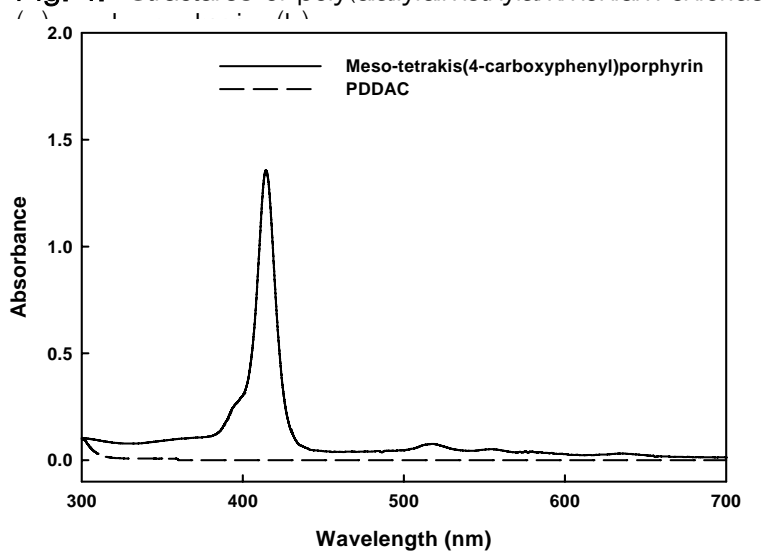

Fig. 2. UV-Vis absorption spectra of PDDAC and meso-tetrakis(4-carboxyphenyl)porphyrin solution.

| 24 | www.ksdf.or.kr
The structures of PDDAC and porphyrin were shown in Fig. 1. In the first step, a negatively charged cotton fibers was then immersed into PDDAC aqueous solution $(0.2 \mathrm{~g} / 200 \mathrm{ml})$ for 20min. After 20min, PDDAC layer on cotton fibers was dried with nitrogen gas. In this process, PDDAC layer having positive charge was attracted on the negatively charged cotton fibers, which occurred by ionic attraction forces. In the second step, the deposited PDDAC layer was then transferred into porphyrin aqueous solution $(0.03 \mathrm{~g} / 200 \mathrm{ml})$ for $20 \mathrm{~min}$. After rinsing in distilled water, porphyrin layer on the cotton fibers was dried with nitrogen gas.

\subsection{Measurements}

Reflectance measurements on the dyed fibers were carried out using a Datacolor SF 600 plus spectrophotometer interfaced to a PC. Measurements were taken with the specula component of the light excluded and the UV component included, using illuminant D65 and $10^{\circ}$ standard observer.

The average of three reflectance measurements, taken at different positions on the dyed fiber substrates, was carried out. Color strength $(\mathrm{K} / \mathrm{S})$ was calculated by the Kubelka-Munk formula.

$$
\mathrm{K} / \mathrm{S}=\frac{(1-\mathrm{R})^{2}}{2 \mathrm{R}}
$$

Where, $\mathrm{K}, \mathrm{S}$ and $\mathrm{R}$ are the coefficient of absorption, scattering of the dye $\lambda$ and the reflected light at wavelength respectively at $\max$.

\section{Results and discussion}

Fabrication for self-assembly coloration approach using PDDAC and meso-tetrakis(4-carboxyphenyl)porphyrin are shown in Fig. 3. These self-assembly multi-layers were deposited sequentially until the desired numbers of multi-layers were achieved.

Fig. 3 shows a schematic illustration of self-assembly fabrication for coloration approach between PDDAC and porphyrin dye. In step A, a negatively charged cotton fiber surface was immersed in the solution of the positively charged PDDAC. Self-assembly attraction of the first polyelectrolyte monolayer occurred at this step. 

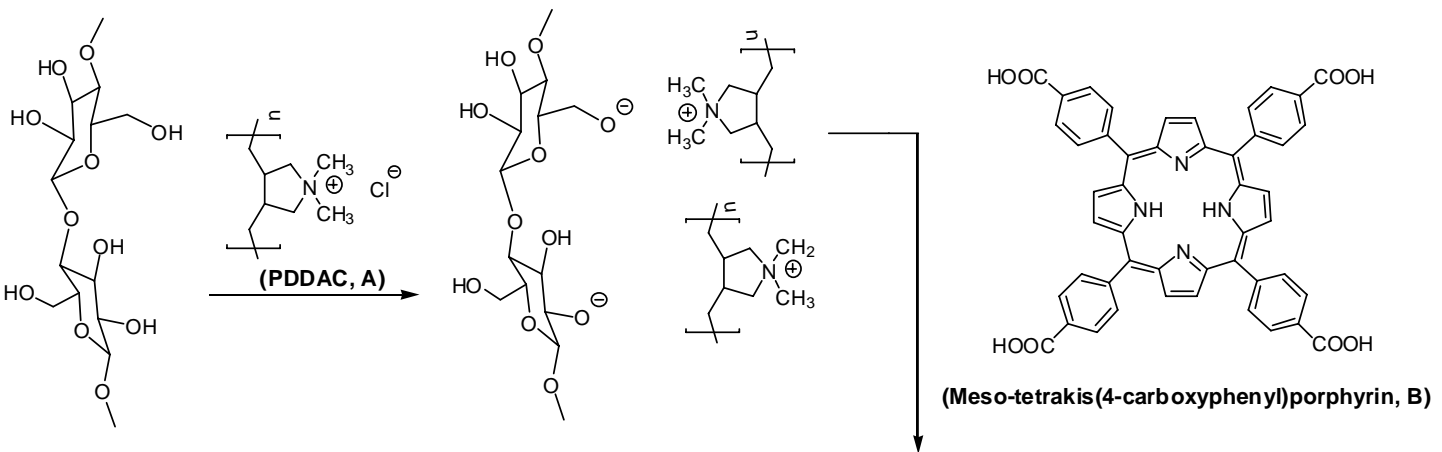

(Cellulosic polymer)

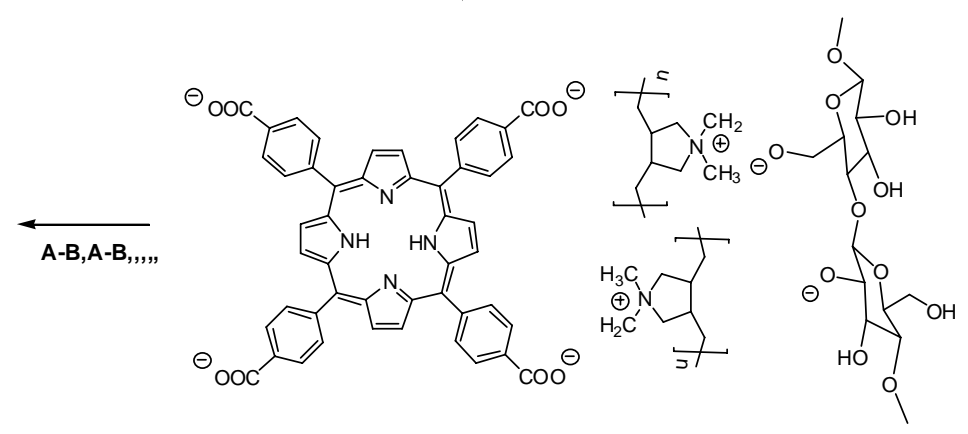

Fig. 3. Scheme of the self-assembly coloring fabrication using PDDAC/meso-tetrakis(4-carboxyphenyl) porphyrin.

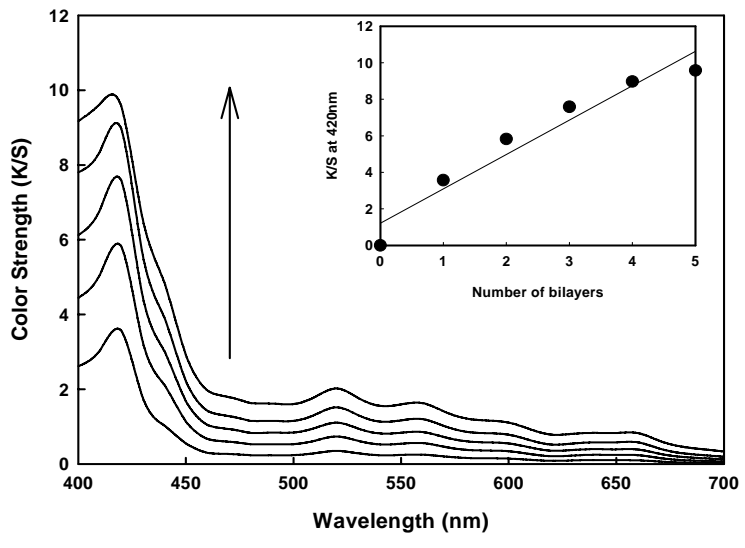

Fig. 4. K/S spectra of the PDDAC and porphyrin bilayers.

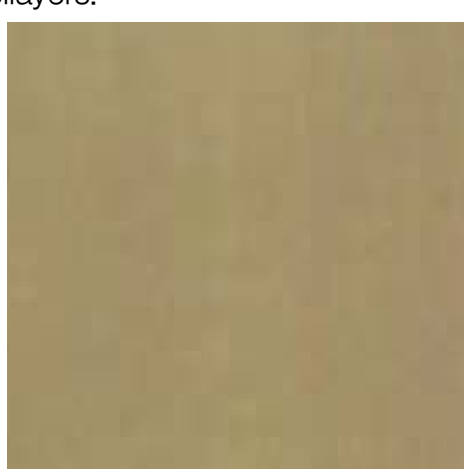

(a)

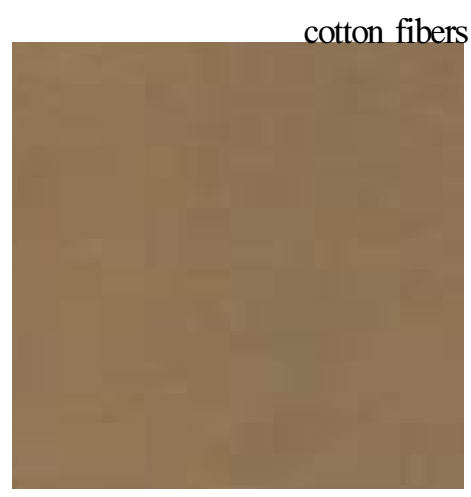

(b)
In step B, the polyelectrolyte monolayer was immersed into the negatively charged porphyrin dye aqueous solution. The self-assembly attraction and corresponding coloration between PDDAC and porphyrin caused by electrostatic forces were formed on the cotton fiber surface. This self-assembly fabrication on the cellulosic substrate was sequentially deposited until the desired number of multilayers were achieved.

In this study, the growth of porphyrin dye layers on cotton fibers formed by self-assembly LbL method was determined with the K/S spectra. In Fig. 4, the K/S spectra of the PDDAC and porphyrin dye multilayers on cotton fibers showed that the color strength of porphyrin

Fig. 5. The coloration images of the PDDAC and porphyrin dye layers: (a) 1 layer, (b) 3 layers, (c) 5 layers. 
increased with increasing the bilayer numbers. The inset shows that the linear increase in K/S values at $420 \mathrm{~nm}$ with increasing the bilayer numbers indicates an appropriate deposition accomplishment with almost equal amount of PDDAC and porphyrin dye in the each cycle.

In Fig. 5, the coloration images of the fabricated layers show that the deeper coloration was obtained with increasing the bilayer numbers. From the results, it can be proposed that the fabricated multilayer coloring deposition with positively charged PDDAC and negatively charged porphyrin dye was successfully achieved. In this context, the purpose of this preliminary work is to attempt and study the direct coloring deposition on cotton fibers using positively and negatively charged molecules. It can be assumed that this is very interesting coloration approach using electrostatic self-assembly attraction. In addition, for the practical view point, the fastness properties should be tested and compared to conventional batch dyeing methods. This further experiment will be determined.

\section{Conclusion}

The self-assembly multilayer films using positive charged PDDAC and negative charged porphyrin dye were prepared on the cotton fibers. Multi-layer thin dye films have been successfully prepared by this electrostatic self-assembly method. Coloration point of the fabricated multilayers have been measured the level of color strength $(\mathrm{K} / \mathrm{S})$. The $\mathrm{K} / \mathrm{S}$ spectra of the fabricated layers showed gradual increase behaviors.

\section{Acknowledgements}

This research was financially supporting by the Ministry of Education, Science Technology (MEST) and Korea Industrial Technology Foundation (KOTEF) through the Human Resource Training Project for Regional Innovation.

\section{References}

1. Guojun Z, Haihong Y, Shulan J, Zhongzhou L, Self-assembly of polyelectrolyte multilayer pervaporation membranes by a dynamic layer-by-layer technique on a hydrolyzed polyacrylonitrile ultrafiltration | 26 | www.ksdf.or.kr membrane, Journal of Membrance Science, 292, 1-8(2007).

2. Tsetska R, Kamelia K, Polypeptide multilayer films on colloidal particles: An in situ electro-optical study, Journal of Colloid and Interface Science, 308, 309-317(2007).

3. Yi L, Tianhong C, Ion-sensitive field-effect transistor based $\mathrm{pH}$ sensors using nano self-assembled polyelectrolyte/nanoparticle multilayer films, Sensors and Actuators B, 123, 148-152(2007).

4. Kim SH, Shin CJ, Keum SR, Koh K, Layer-by-layer self-assembled multilayer of cationic spiroxazine and polystyrenesulfonate, Dyes and Pigments, 72, 378-382(2007).

5. Son YA, Park YM, Shin CJ, Kim SH, Self-assembly multi-layer of diazonium resin and its coupling reaction with J-acid and $\mathrm{H}$-acid, Dyes and Pigments, 72, 345-348(2007).

6. Kim SH, Yu CY, Shin CJ, Choi MS, Photochromic layer-by-layer films of spiroxazine polymer, Dyes and Pigments, 75, 250-252(2007).

7. Ozoemena KI, Nyokong T, Electrocatalytic oxidation and detection of hydrazine at gold electrode modified with iron phthalocyanine complex linked to mercaptopyridine self-assembled monolayer, Talanta, 67, 162-168(2005).

8. Katsumi T, Photochemical and photophysical behaviour of porphyrins and phthalocyanines irradiated with violet or ultraviolet light, Journal of Porphrins and Phthalocyanines, 5, 77-86(2001).

9. Punidha S, Ravikanth M, Synthesis of covalently linked linear porphyrin triad and tetrad containing different porphyrin sub-units, Tetrahedron, 64, 80168028(2008).

10. Caminos DA, Durantini EN, Interaction and photodynamic activity of cationic porphyrin derivatives bearing different patterns of charge distribution with GMP and DNA, Journal of Photochemistry and Photobiology A; Chemistry, 198, 274-281(2008).

11. Lu X, Yuan H, Zuo G, Yang J, Study of the size and separation of pinholes in the self-assembled thiol-porphyrin monolayers on gold electrodes, Thin Solid Films, 516, 6476-6482(2008).

12. Katsuhiko T, Takashi N, Preparation of dye thin films on fibers by electrostatic layer-by-layer 
adsorption, ISDF proceeding, 149-150(2006).

13. Guillaume G, Vincent S, Francois L, Robert G, Michel G, Yves C, Pierre K, Synthesis and photo cytotoxic activity of new chlōin polyamine conjugates, Bioorganic \& Medicinal Chemical Letters, 16, 31883192(2006). 